\title{
Tuberculosis con resistencia a rifampicina en Chile
}

\author{
CARLOS PEÑA M.* y NADIA ESCOBAR S.**
}

\section{Tuberculosis with resistance to rifampicin in Chile}

The high success rate of shortened Tuberculosis (TB) treatments has been achieved by the association of bactericidal and sterilizing drugs. The main drugs are Rifampicin and Isoniazide. When Rifampicin cannot be used by resistance, the treatment is prolonged and success in healing is significantly reduced. Resistance to Rifampicin is often accompanied by resistance to Isoniazide (Multidrug resistance or MDR). WHO reports that only $44 \%$ of estimated TB cases with resistance to Rifampicin were diagnosed in 2019 (465,000 cases projected) and only 38\% of estimated cases were treated, with a large proportion of cases remaining undiagnosed and untreated. In Chile, monitoring of susceptibility to first-line drugs is conducted in strains of Mycobacterium tuberculosis by molecular biology since 2014, observing a progressive increase in cases with Rifampicin resistance from 1\% for that year (23 cases) to 2.2\% in 2019 (65 cases). Most cases of resistance to Rifampicin correspond to cases of initial resistance. In cases with resistance to Rifampicin we carry out susceptibility study to second-line drugs in a national reference laboratory. MDR-TB therapy has low efficacy, with frequent abandonments for its long therapy time and toxicity. New non-injectable treatments and use of Clofazimine, Fluorquinolones, Linezolid and Bedaquiline are achieving a better cure rate. Recently, Chile's TB Control Program has this most effective and shorter-lasting oral therapy with these drugs.

Key words: Rifampicin resistant tuberculosis; Multidrug-resistant treatment.

\section{Resumen}

El éxito de los tratamientos acortados de la tuberculosis se debe a la asociación de fármacos bactericidas y esterilizantes, principalmente Rifampicina e Isoniazida. Cuando la Rifampicina no puede ser utilizada por resistencia, los tratamientos son más prolongados y el éxito en la curación se reduce. La resistencia a Rifampicina frecuentemente se acompaña de resistencia a Isoniazida (Multidrogoresistencia o MDR). La OMS informa que en 2019 se diagnosticaron sólo el 44\% de los casos estimados de tuberculosis con resistencia a Rifampicina (se proyectaba 465.000 casos) y se trató sólo al 38\% de los casos estimados, quedando una gran proporción de casos sin diagnosticar y sin tratar. En Chile la vigilancia de la susceptibilidad a fármacos de primera línea en cepas de Mycobacterium tuberculosis se efectúa mediante biología molecular desde 2014, observándose un progresivo incremento de casos resistentes a Rifampicina desde 1\% (23 casos) para ese año hasta 2,2\% (65 casos) en 2019. La mayoría de casos de resistencia a Rifampicina corresponden a resistencia inicial. En casos con resistencia a Rifampicina realizamos estudio de susceptibilidad a fármacos de segunda línea en el laboratorio de referencia nacional. La terapia de TB-MDR tradicional tiene baja eficacia, con abandonos frecuentes por su largo tiempo de terapia y toxicidad. Nuevos tratamientos sin inyectables y el uso de Clofazimina, Fluorquinolonas, Linezolid y Bedaquilina tienen una mejor tasa de curación. Recientemente, el Programa de Control de la Tuberculosis de Chile dispone de esta terapia más eficaz y de menor duración por vía oral con estos fármacos.

Palabras clave: Tuberculosis resistente a rifampicina; tratamiento de la tuberculosis multidrogoresistente.

* Encargado Programa de Control y eliminación de la Tuberculosis (PROCET) del Servicio de Salud Metropolitano Central. Hospital Clínico San Borja Arriarán.

** Directora Nacional del PROCET. Ministerio de Salud de Chile. 


\section{Resistencia a fármacos de Primera línea: Multidrogoresistencia, el legado de la Rifampicina}

La rifampicina $(\mathrm{R})$ tiene un papel muy crucial en los tratamientos acortados de la tuberculosis, descubierta en 1957 y utilizada a partir del año 1966 es un fármaco bactericida dosis-dependiente y además esterilizante que asociado a Pirazinamida es capaz de acortar el tiempo de terapia ${ }^{1}$. Los regímenes terapéuticos de la década de los 70 que utilizaban $\mathrm{R}$ en dosis de $600 \mathrm{mg}$ diarios logran un éxito terapéutico superior al $95 \%{ }^{2}$. Como contraparte a raíz de su alto potencial inductor de actividad de citocromo P450 hepático es capaz de reducir los niveles plasmáticos y en consecuencia los efectos clínicos de innumerables fármacos como los Inhibidores de Proteasas utilizados en la terapia de infección por $\mathrm{VIH}^{3}$. Su nivel sanguíneo terapéutico mínimo es de $8 \mu \mathrm{g} / \mathrm{ml}^{4}$. El nivel sanguíneo máximo del fármaco se obtiene entre las 2-4 h desde su ingesta oral. Su penetración a las meninges inflamadas es del $10 \%$ del nivel sanguíneo por lo que se requiere obtener una concentración sanguínea máxima (Cmax) de 22 $\mu \mathrm{g} / \mathrm{ml}$ o más. Su uso crónico aumenta la depuración del fármaco y podría ocasionar bajos niveles terapéuticos ${ }^{5}$. Existen estudios que señalan que las dosis actuales recomendadas de $10 \mathrm{mg} /$ $\mathrm{kg}$ /día podrían ser insuficientes para lograr una Cmáx adecuada por tiempo suficiente. Por esta razón se han realizado ensayos clínicos con dosis más elevadas (13-40 mg/kg/día) demostrando seguridad y mejor biodisponibilidad del fármaco y probablemente una capacidad esterilizante más rápida ${ }^{6,7,8,9,10}$. Sin embargo, estas situaciones son difíciles de evaluar sin disponer de niveles plasmáticos. Existen condiciones clínicas como la diabetes, la infección por VIH y los cuadros de diarrea persistente, que podrían ocasionar una mala absorción del fármaco y por lo tanto ocasionar bajos niveles plasmáticos, lo que indicaría la necesidad de usar dosis mayores $4,7,11,12,13,14$. Indudablemente la $\mathrm{R}$ continuará siendo hasta el momento un fármaco esencial y probablemente asociado a una fluorquinolona podría demostrar ser útil en terapias más acortadas que las actuales ${ }^{15}$.

La resistencia secundaria a $\mathrm{R}$ se puede generar cuando el fármaco asociado en la terapia, Isoniazida $(\mathrm{H})$, es resistente y no es detectado antes de pasar a la fase de biterapia intermitente, exponiendo al paciente a monoterapia con $\mathrm{R}$ y a una eventual resistencia a la $\mathrm{R}$, transformando al paciente en multidrogoresistente (resistencia a $\mathrm{R}$ y a $\mathrm{H}$ o MDR). Estamos observando además, la presencia de casos con mono resistencia inicial a $\mathrm{R}$ (pacientes vírgenes a tratamiento previo de la tuberculosis) que son considerados desde el punto de vista operacional como un caso MDR. El diagnóstico de MDR habitualmente se genera a través de los resultados de la sensibilidad evaluada en cultivos positivos mediante pruebas fenotípicas, pero las técnicas moleculares que detectan genes con mutaciones que confieren resistencia, especialmente a $\mathrm{R}$ (mutación en gen rpoB en al menos el $96 \%$ de los casos) a través de hibridación reversa (Genotype o LPAs) o amplificación del ADN por métodos enzimáticos (polimerasa en cadena o PCR) son cada vez más utilizadas ${ }^{16,17}$.

\section{Situación mundial actual de la tuberculosis multidrogoresistente}

La OMS ha considerado a la TB-MDR un problema de salud pública importante y el reporte del año 2020 estima que en el año 2019 existían 465.000 personas con resistencia a $\mathrm{R}$ en el mundo, de las cuales el $78 \%$ son TB-MDR. Los casos se concentran en India (27\%), China (14\%) y la Federación Rusa (8\%). Sólo en el 61\% de los casos de tuberculosis bacteriológicamente demostrados se realizan exámenes de testeo de resistencia a R. Aproximadamente el 3,3\% de los casos nuevos y el $17,7 \%$ de los casos antes tratados de tuberculosis muestran resistencia a R. Desafortunadamente, sólo se notificaron 206.030 casos (44\%) y de estos casos 177.099 iniciaron tratamiento. Esta situación muestra una importante brecha para el adecuado tratamiento de los casos estimados lo que mantiene una importante carga de casos no diagnosticados y no tratados en la comunidad ${ }^{18}$.

\section{Terapias para TB-MDR en el mundo}

La aparición de casos de TB-MDR se puede deber al uso inapropiado de los fármacos de primera línea por parte de los equipos sanitarios, a la mala calidad de los fármacos y a la mala adherencia a la terapia o al abandono de la misma por parte del paciente. Existen muchas causas de esta baja adherencia: el paciente ya se siente sano y no desea continuar su terapia, puede abandonar por la aparición de efectos colaterales o eventos adversos a los fármacos, puede tener dificultades en la relación con los proveedores de salud. Además, el paciente puede tener problemas de salud mental o adicciones, problemas de transporte a los centros de terapia o dificultades laborales 
que deben ser intervenidas por equipos multidisciplinarios. Por otra parte, las terapias en casos de resistencia a $\mathrm{R}$ solían ser muy prolongadas $\mathrm{y}$ tóxicas, con una baja tasa de curación (inferior al $50 \%$ ), con mayor mortalidad y más abandonos (pérdida de seguimiento).

La terapia de la TB-MDR es un desafío para el clínico y la propuesta más recomendada era recurrir al uso de al menos 5 fármacos por al menos 20 meses de uso. La selección de los fármacos a utilizar estaría de acuerdo a la historia previa de uso, a los patrones de resistencia más comunes y a los tests de susceptibilidad a fármacos. Los tratamientos de TB-MDR resultantes basados en regímenes individualizados mostraban una curación de $64 \%$, mientras que los regímenes estandarizados tenían una curación de 53\%. A menudo se presentaban reacciones adversas como náuseas y vómitos en el $32,8 \%$, diarrea en $21 \%$ y pérdida auditiva en el $12 \%{ }^{19}$. Los efectos adversos eran muy frecuentes $(79 \%$ de los pacientes en tratamiento), en el $20 \%$ de ellos se debía cambiar la dosis del fármaco y en $64 \%$ se debía interrumpir su administración ${ }^{20}$. La OMS en el año 2016 recomendó el uso de al menos 4 fármacos, que debían pertenecer a una clasificación de acuerdo a su mayor actividad y potencia, entre estos fármacos destacaban como más útiles la Fluorquinolona y los inyectables como kanamicina, los cuales eran acompañados por un cortejo de otros fármacos en que se incluían Pirazinamida, Etambutol y Etionamida entre otros ${ }^{21}$. Sin embargo, este tratamiento de 8 meses de fase intensiva y 12 meses de fase de continuación, era demasiado prolongado, con alta toxicidad y de eficacia discreta. La aprobación de la OMS de una terapia más acortada a 9-12 meses en el año 2016 (short standardized regimen) fue un hito importante. Este régimen (Bangladesh) utilizando fluorquinolona (gatifloxacino) e isoniazida a altas dosis, asociado a Clofazimina, Protionamida, Etambutol y Kanamicina en la fase inicial de 4 meses mostraba una curación de $87,9 \%{ }^{22}$. Posteriormente, el año 2018, se incorporan como drogas útiles recomendadas, además de las fluorquinolona, a la Bedaquilina, el Linezolid y la Clofazimina, que debían estar presentes en los actuales tratamientos completamente orales (se retiró el uso de los inyectables por su toxicidad vestibular y auditiva) $)^{23,24}$.

Con la incorporación de nuevos fármacos como Bedaquilina, que inhibe la ATP-sintasa de la micobacteria -destruyendo a las micobacterias, independientemente de si se encuentran en replicación -, se ha logrado reducir el tiempo de conversión del cultivo (cultivo sin crecimiento de los bacilos) ya desde las 4 semanas de uso y se ha comprobado una mayor tasa de curación respecto a terapias sin este fármaco ${ }^{25,26}$. También la incorporación de Delamanid que inhibe la síntesis de ácido micólico, ha contribuido a aumentar la proporción de cultivos negativos a los 2 meses de terapia y ha cooperado en reducir la mortalidad incluso en TBC extensamente resistente o XDR (con resistencia a fluorquinolona e inyectables), sin mayor incidencia de síncopes asociados a su efecto de prolongación del intervalo QT que se presenta en el ECG en el 3,8\% de los pacientes que usan dosis altas del fármaco $(200 \mathrm{mg})^{27,28,29}$.

\section{La resistencia a Rifampicina en Chile}

Nuestro país a través de su laboratorio de referencia nacional, mantiene desde hace varias décadas un sistema de vigilancia permanente de la susceptibilidad a fármacos que muestra una baja proporción de casos resistentes, menores a $1 \%$ para la resistencia a $\mathrm{R}$. El número de casos de tuberculosis resistentes a $\mathrm{R}$ se ha ido incrementando en el tiempo, el promedio de casos entre el año 2000 y 2015 era de 14 casos anuales y entre el año 2016 y 2017 este promedio anual se duplicó, llegando a 30 casos anuales. Para los años 2018 y 2019 este aumento es mayor llegando a un promedio anual de $72 \operatorname{casos}^{30}$ (Figura 1).

Desde el año 2014 la vigilancia de la resistencia se efectúa a través de pruebas de biología molecular, registrando desde entonces la presencia de una resistencia a $\mathrm{R}$ cercana a $1 \%$, pero a partir del año 2018 esta resistencia se duplica ya que se detecta en cerca del $2 \%$ de los casos totales de tuberculosis (69 casos), situación que se mantiene el año 2019 (65 casos) ${ }^{30}$ (Figura 2).

Esta situación ha sido un apoyo más para incorporar masivamente un testeo inicial de la resistencia a $\mathrm{R}$, en todos los pacientes con tuberculosis bacteriológicamente demostrada -la mayoría son casos pulmonares-, mediante biología molecular, con técnicas de PCR entre las que destacan el GeneXpert MTB/RIF, una plataforma automatizada, en tiempo real y semicuantitativa, que tiene una sensibilidad de detección de resistencia a $\mathrm{R}$ de alrededor de $95 \%$ y una especificidad de $98 \%$.

\section{Tratamiento de la TB-MDR en Chile}

Nuestro país tradicionalmente presentaba un muy bajo número de casos de TB-MDR y la mayoría procedía de fracasos por mal cumplimiento 
Figura 1. Casos de Tuberculosis con resistencia a rifampicina. Chile, 2000-2019. Las cifras de la ordenada y de cada una de las columnas señala el número de casos de tuberculosis con resistencia a rifampicina.

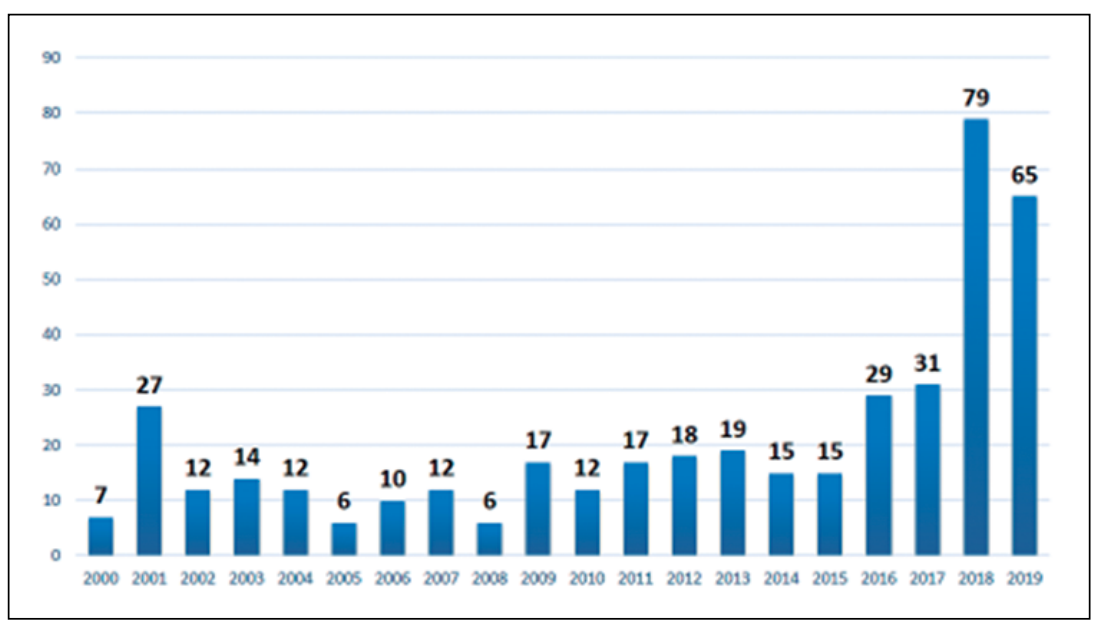

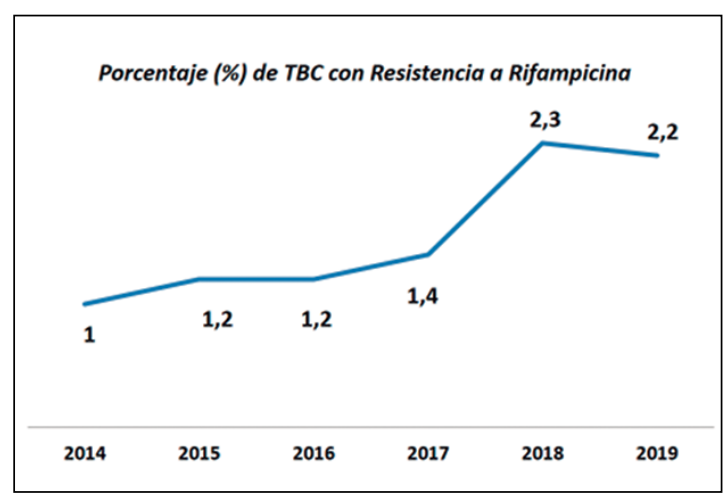

Figura 2. Proporción de casos de Tuberculosis de todas las localizaciones con resistencia a rifampicina. Chile (2014-2019). Las cifras que acompañan la curva representan los porcentajes $(\%)$ de casos.

de la terapia, especialmente abandonos múltiples. Para el tratamiento de estos casos se utilizaban los fármacos inyectables, como Kanamicina asociado a fármacos de baja potencia bactericida y escaso poder esterilizante como ácido paraaminosalicílico (PAS), Etionamida y Cicloserina, además de la adición de Etambutol y Pirazinamida. Los resultados de estos tratamientos mostraban una baja capacidad de curación. El promedio de éxito terapéutico entre los años 1997 y 2010 era de $36 \%$. Posteriormente se utilizaron esquemas con adición de fluorquinolona (FQNs), pero siempre asociados a la matriz de una terapia con inyectables y más tarde se agregó Clofazimina. A pesar de la tendencia a una mayor curación, los resultados no eran óptimos. El promedio de curación entre los años 2011 y 2017 es de 44\%, pero si observamos el promedio entre los años 2010 y
2015 la curación solo se obtenía en el 40\% de los casos. Entre los años 2016 y 2017 (últimos años de evaluación y esquema "shorter"), el promedio de curación aumenta a $59,7 \% \%^{30}$ (Figura 3 ). Los casos actuales con resistencia a $\mathrm{R}$ en una alta proporción son casos nuevos (resistencia inicial) y no fracasos de tratamientos.

A partir del mes de octubre del año 2020 la terapia de la TB-MDR en Chile utiliza un esquema de 6 meses diarios con Bedaquilina, Linezolid, Moxifloxacino y Clofazimina seguido de 3 meses con Moxifloxacino y Clofazimina ${ }^{31}$. Estos fármacos son entregados por el PROCET del nivel central, luego de evaluar los casos con un comité técnico de especialistas. De esta manera nuestro país queda en la vanguardia de las terapias recomendadas internacionalmente con un mayor potencial de éxito. Estos fármacos tienen diferentes mecanismos de acción y pueden ocasionar algunas reacciones adversas que deben ser monitoreadas (Tabla 1).

\section{El camino operativo para el control y manejo de la resistencia a fármacos}

Para enfrentar en forma integral el problema de la resistencia a $\mathrm{R}$, y en general el manejo de la tuberculosis, es necesario desarrollar un plan colaborativo, que involucra tanto a los pacientes como a los equipos de salud que proveen los cuidados en estos casos. Una permanente capacitación, asesoría y supervisión del personal sanitario son materia esencial para la aplicación de este plan. En términos generales, buscamos potenciar la prevención de las resistencias a los fármacos, a través del apropiado uso de estos en 


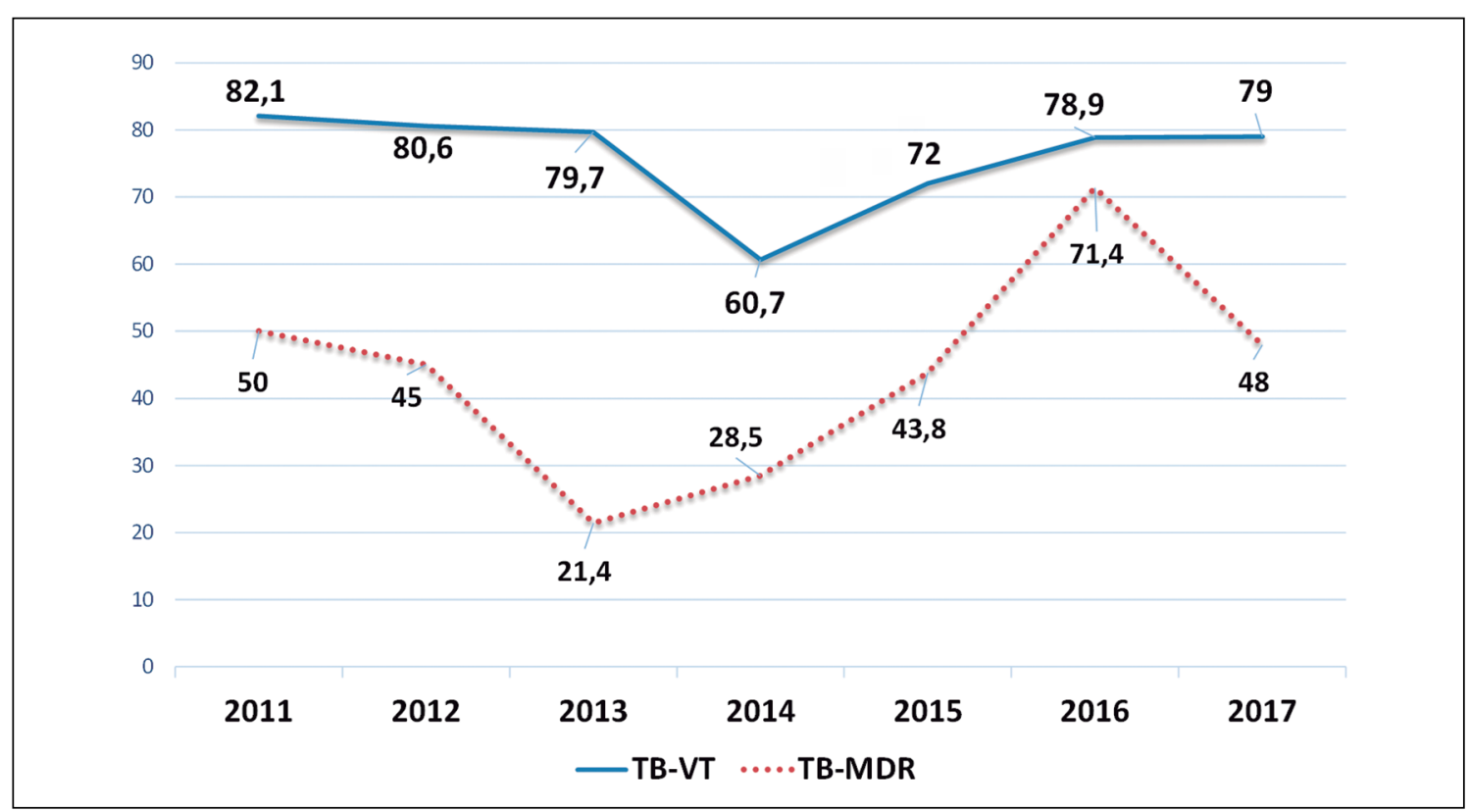

Figura 3. Proporción de éxito de terapia en cohorte de casos nuevos de Tuberculosis (TB-VT) y Tuberculosis multidrogo-resistente (TB-MDR). Chile (2011-2017). Las cifras de la ordenada y las que acompañan a las curvas representan porcentajes (\%) de curación.

Tabla 1. Propiedades de los fármacos usados en el tratamiento de la Tuberculosis Multidrogo-resistente

\begin{tabular}{|c|c|c|c|c|c|c|}
\hline Fármaco & Moxifloxacino & Bedaquilina & Delamanid & Linezolid & Clofazimina & Cicloserina \\
\hline $\begin{array}{l}\text { Mecanismo } \\
\text { de acción }\end{array}$ & $\begin{array}{c}\text { Bactericida y } \\
\text { Esterilizante } \\
\text { Inhibe ADN } \\
\text { Girasa }\end{array}$ & $\begin{array}{c}\text { Bactericida } \\
\text { Inhibe } \\
\text { AT-sintasa }\end{array}$ & $\begin{array}{l}\text { Bactericida } \\
\text { Inhibe síntesis } \\
\text { ácidos } \\
\text { micólicos }\end{array}$ & $\begin{array}{c}\text { Bactericida } \\
\text { Inhibe síntesis } \\
\text { proteica }\end{array}$ & $\begin{array}{l}\text { Bactericida } \\
\text { Inhibe } \\
\text { replicación } \\
\text { ADN y } \\
\text { respiración } \\
\text { celular }\end{array}$ & $\begin{array}{l}\text { Bacteriostático } \\
\text { Inhibe } \\
\text { formación } \\
\text { péptidoglicano } \\
\text { de pared } \\
\text { celular }\end{array}$ \\
\hline $\begin{array}{l}\text { Efectos } \\
\text { adversos }\end{array}$ & $\begin{array}{c}\text { Prolonga QT } \\
\text { Tendinitis }\end{array}$ & $\begin{array}{c}\text { Prolonga QT } \\
\text { Hepatitis }\end{array}$ & $\begin{array}{l}\text { Prolonga QT } \\
\text { Interacción } \\
\text { con fármacos }\end{array}$ & $\begin{array}{l}\text { Toxicidad } \\
\text { hematológica } \\
\text { Neuropatía }\end{array}$ & $\begin{array}{c}\text { Prolonga QT } \\
\text { Pigmentación } \\
\text { cutánea }\end{array}$ & $\begin{array}{c}\text { Psicosis } \\
\text { Depresión } \\
\text { Convulsiones }\end{array}$ \\
\hline $\begin{array}{l}\text { Presentación } \\
\text { (comprimidos) }\end{array}$ & $400 \mathrm{mg}$ & $100 \mathrm{mg}$ & $50 \mathrm{mg}$ & $600 \mathrm{mg}$ & $100 \mathrm{mg}$ & $250 \mathrm{mg}$ \\
\hline Dosis habitual & 400 mg/día & $\begin{array}{c}400 \mathrm{mg} / \text { día } \mathrm{x} \\
2 \mathrm{sem} \text { y luego } \\
200 \mathrm{mg} \mathrm{c} / 48 \mathrm{~h} \\
\text { x } 22 \mathrm{sem}\end{array}$ & $\begin{array}{l}100 \mathrm{mg} \mathrm{c} / 12 \mathrm{~h} \\
\text { x } 24 \mathrm{sem}\end{array}$ & 600 mg/día & 100 mg/día & 750 mg/día \\
\hline
\end{tabular}

los casos sensibles, fortaleciendo la adherencia a los tratamientos vigilados mediante la estrategia DOTS (Directly Observed Therapy-short course) como uno de los ejes principales.

Para lograr además buenos resultados con la terapia es crucial lograr un diagnóstico precoz de la resistencia. Con el apoyo de la biología molecular, en plataformas de PCR como el GeneXpert $M T B / R I F$, es posible conocer la sen- sibilidad inicial a $\mathrm{R}$ en las muestras directas de expectoración o de tejidos y fluidos que resultan positivas y de esta manera instalar un tratamiento adecuado. No sólo podemos diagnosticar una resistencia a $\mathrm{R}$, también existe un cartucho para PCR de GeneXpert XDR, que detecta mutaciones de genes que confieren resistencia a Isoniazida (genes promotor InhA, KatG, fabG1 y región intergénica oxyR-ahpC), FQNs (genes Girasa A 
y B), Etionamida (promotor InhA) e inyectables como Amikacina, Kanamicina (gen rrs) y Capreomicina (promotor eis). Esta tecnología tiene buena sensibilidad para detectar estas mutaciones: H 91,4-98,8\%, FQN 93,1-93,3\%, Amikacina 91,9-96,4\% y Kanamicina 87,9-96,7\% según se compare con estudio fenotípicos o secuenciación del genoma ${ }^{32}$. Este cartucho no detecta resistencia a $\mathrm{R}$ y se utiliza luego de realizar el diagnóstico de la tuberculosis mediante el cartucho GeneXpert $M T B / R I F$ que nos informa la presencia o ausencia de resistencia a $\mathrm{R}$.

Para el tratamiento de los casos con resistencia a $\mathrm{R}$ se dispone actualmente de regímenes totalmente orales, sin los inyectables que producían trastornos vestibulares y auditivos y por supuesto dolor en su administración, durante por lo menos los 4 meses diarios en que suele utilizarse. Los actuales fármacos tienen una mejor tolerancia digestiva, ya que existen regímenes sin Etionamida,que es el fármaco que ocasionaba mayor rechazo por molestias digestivas en estos pacientes. Los antiguos tratamientos tenían una duración de 18 a 20 meses o más. Actualmente la OMS promueve el uso de nuevos fármacos, que combinados tienen una mayor eficacia, pero que deben ser muy controlados, de ahí la necesidad de capacitación de nuestros equipos técnicos ${ }^{33}$. Es posible lograr la curación con esquemas de sólo 9 a 11 meses utilizando Bedaquilina, Levofloxacino (preferido por menor efecto sobre el intervalo QT del ECG) o Moxifloxacino, Linezolid y Clofazimina. La duración del esquema en todo caso dependerá del momento de negativización del cultivo. Investigaciones operacionales en curso nos informarán de terapias más acortadas, incluso de 6 meses, un sueño hasta hace poco tiempo atrás. Algunos de los nuevos fármacos como Bedaquilina se están evaluando incluso en esquemas acortados para tuberculosis sensible asociado a Pretomanid y Pirazinamida mostrando mayor actividad bactericida que la asociación de fármacos del esquema primario $(\mathrm{H}, \mathrm{R}, \mathrm{E}, \mathrm{Z})$ pero se observaron hasta $8 \%$ de casos de toxicidad hepática grave con el sólo uso durante 2 meses $^{34}$, de manera que aún debemos esperar estudios que ofrezcan mayor seguridad clínica.

\section{Conclusiones}

La resistencia a $\mathrm{R}$ se está transformando en un problema de salud pública en incremento mundial. Se debe mantener una permanente vigilancia de la susceptibilidad de las cepas circulantes en la comunidad. En nuestro país, el laboratorio nacio- nal de referencia desarrolla esta trazabilidad con tecnologías más complejas, como sondas en línea y secuenciaciones genéticas, además de testeos fenotípicos de susceptibilidad a fármacos con diferentes dosis para colaborar en las decisiones de la terapia en cada caso clínico. Es muy necesario el diagnóstico oportuno de esta condición bacteriológica, mediante el uso de técnicas de biología molecular, en las muestras de expectoración de los casos de tuberculosis pulmonar, especialmente, y en forma muy precoz, ya que la mayor proporción de casos resistentes corresponden a una resistencia inicial (casos nuevos nunca tratados) y no a fracasos de terapia. Para los servicios de salud los métodos de amplificación del ADN de la micobacteria como GeneXpert MTB/RIF son actualmente bastante accesibles, tienen una buena sensibilidad y son confiables y seguros. Existe una revolución en las actuales terapias de la TBMDR con fármacos menos tóxicos, de mayor potencia bactericida y esterilizante en esquemas de uso completamente oral. Estas terapias tienen alta eficacia y aceptabilidad en los pacientes. Nuestro país cuenta actualmente con las mejoras en el diagnóstico y tratamiento recomendados por la OMS y expertos mundiales. Además es altamente necesario implementar todas las medidas que aseguren la adherencia del paciente al tratamiento junto con mantener una constante capacitación de los equipos técnicos de tuberculosis para el adecuado manejo y control de las terapias con estos nuevos fármacos.

\section{Bibliografía}

1.- EAST AFRICAN/MEDICAL RESEARCH COUNCILS. Controlled clinical trial of short-course (6-month) regimens of chemotherapy for treatment of pulmonary tuberculosis. Lancet 1972; 1 (7760): 1079-85. Disponible en: https://doi.org/10.1016/S01406736(72)91426-2

2.- HONG KONG/BRITISH MEDICAL RESEARCH COUNCIL. Controlled trial of 6-month and 8-month regimens in the treatment of pulmonary tuberculosis: The results up to 24 months. Tubercle. 1979; 60 (4): 979- 201-0.

3.- JUSTESEN US, ANDERSEN AB, KLITGAARD NA, BRØSEN K, GERSTOFT J, PEDERSEN C. Pharmacokinetic interaction between rifampin and the combination of indinavir and low-dose ritonavir in HIVinfected patients. Clin Infect Dis. 2004; 38 (3): 426-9. doi: 10.1086/380794.

4.- GURUMURTHY P, RAMACHANDRAN G, KUMAR H, RAJASEKARAN S, PADMAPRIYADARSINI C, SWAMINATHAN S, et al. Malabsorption of 
rifampin and isoniazid in HIV-infected patients with and without tuberculosis. Clin Infect Dis. 2004; 38 (2): 280-3. doi: 10.1086/380795

5.- ISRAILI ZH, ROGERS CM, EL-ATTAR H. Pharmacokinetics of antituberculosis drugs in patients. J Clin Pharmacol. 1987; 27 (1): 78-83. doi: 10.1177/009127008702700113.

6.- SVENSSON RJ, NIWARD K, FORSMAN LD, BRUCHFELD J, PAUES J, ELIASSON E, et al. Individualised dosing algorithm and personalised treatment of high-dose rifampicin for tuberculosis. $\mathrm{Br} \mathrm{J}$ Clin Pharmacol. 2019; 85 (10):2341-2350. doi: 10.1111/ bcp. 14048 .

7.- RUSLAMI R, NIJLAND HMJ, ALISJAHBANA B, PARWATI I, VAN CREVEL R, AARNOUTSE RE. Pharmacokinetics and tolerability of a higher rifampin dose versus the standard dose in pulmonary tuberculosis patients. Antimicrob Agents Chemother. 2007; 51 (7): 2546-51. doi: 10.1128/AAC.01550-06.

8.- BOEREE MJ, DIACON AH, DAWSON R, NARUNSKY K, DU BOIS J, VENTER A, et al (PanACEA Consortium). A dose-ranging trial to optimize the dose of rifampin in the treatment of tuberculosis. Am J Respir Crit Care Med. 2015; 191 (9): 1058-65. doi: 10.1164/rccm.201407-1264OC.

9.- AARNOUTSE RE, KIBIKI GS, REITHER K, SEMVUA H, HARAKA F, MTABHO CM, et al. (PanACEA Consortium). Pharmacokinetics, Tolerability, and Bacteriological Response of Rifampin Administered at 600, 900, and 1,200 Milligrams Daily in Patients with Pulmonary Tuberculosis. Antimicrob Agents Chemother. 2017; 24; 61 (11): e01054-17. doi: 10.1128/ AAC.01054-17.

10.- STEINGART KR, JOTBLAD S, ROBSKY K, DECK D, HOPEWELL PC, HUANG D, et al. Higher-dose rifampin for the treatment of pulmonary tuberculosis: a systematic review. Int J Tuberc Lung Dis. 2011; 15 (3): 305-16.

11.- MCILLERON H, WASH P, BURGER A, NORMAN J, FOLB PI, SMITH P. Determinants of rifampin, isoniazid, pyrazinamide, and ethambutol pharmacokinetics in a cohort of tuberculosis patients. Antimicrob Agents Chemother. 2006; 50 (4): 1170-7. doi: 10.1128/ AAC.50.4.1170-1177.2006.

12.- GURUMURTHY P, RAMACHANDRAN G, KUMAR AKH, RAJASEKARAN S, PADMAPRIYADARSINI C, SWAMINATHAN S, et al. Decreased bioavailability of rifampin and other antituberculosis drugs in patients with advanced human immunodeficiency virus disease. Clinical Trial Antimicrob Agents Chemother. 2004; 48 (11): 4473-5. doi: 10.1128/ AAC.48.11.4473-4475.2004.

13.- ALSULTAN A, PELOQUIN CA. Therapeutic drug monitoring in the treatment of tuberculosis: an update. Drugs. 2014; 74 (8): 839-54. doi: 10.1007/s40265-0140222-8.
14.- YUHUI XU, JIANAN WU, SHA LIAO, ZHAOGANG SUN. Treating tuberculosis with high doses of anti-TB drugs: mechanisms and outcomes. Ann Clin Microbiol Antimicrob. 2017; 16 (1): 67. doi: 10.1186/ s12941-017-0239-4.

15.- KWONJUNE J SEUNG, SALMAAN KESHAVJEE, MICHAEL L RICH. Multidrug-Resistant Tuberculosis and Extensively Drug-Resistant Tuberculosis. Cold Spring Harb Perspect Med. 2015; 5 (9): a017863. doi: 10.1101/cshperspect.a017863.

16.- MACLEAN E, KOHLI M, WEBER SF, SURESH A, SCHUMACHER SG, DENKINGER CM, et al. Advances in Molecular Diagnosis of Tuberculosis. Journal of Clinical Microbiology. 2020; 58 (10): e01582-19 DOI: 10.1128/JCM.01582-19.

17.- RENDON A, CENTIS R, D'AMBROSIO L, MIGLIORI GB. WHO Strategies for the Management of Drug-Resistant Tuberculosis. Arch Bronconeumol. 2017; 53 (3): 95-7. doi: 10.1016/j.arbr.2016.07.027.

18.- WHO. Global tuberculosis report 2020. TB disease burden (chapter 4): 23-69 and TB diagnosis and treatment (chapter 5): 71-112. Geneva: World Health Organization; 2020. Licence: CC BY-NC-SA 3.0 IGO.

19.- NATHANSON E, GUPTA R, HUAMANI P, PASECHNIKOV AD, TUPASI TE, VINK K, et al. Adverse events in the treatment of multidrug-resistant tuberculosis: Results from the DOTS-Plus initiative. Int J Tuberc Lung Dis. 2004; 8: 1382-4.

20.- BLOSS E, KUKSA L, HOLTZ TH, RIEKSTINA V, SKRIPCONOKA V, KAMMERER S, et al. Adverse events related to multidrugresistant tuberculosis treatment, Latvia, 2000-2004. Int J Tuberc Lung Dis. 2010; 14: 275-81.

21.- WHO treatment guidelines for drug resistant tuberculosis, 2016 update. World Health Organization. 2016.

22.- VAN DEUN A, MAUG AK, SALIM MA, DAS PK, SARKER MR, DARU P, et al. Short, highly effective, and inexpensive standardized treatment of multidrugresistant tuberculosis. Am J Respir Crit Care Med. 2010; 182: 684-92.

23.- PARK M, SATTA G, KON OM. An update on multidrug-resistant tuberculosis. Clin Med (Lond). 2019; 19 (2): 135-39. doi: 10.7861/clinmedicine.19-2-135.

24.- CAMINERO JA, SCARDIGLI A. Classification of antituberculosis drugs: a new proposal based on the most recent evidence. Eur Respir J. 2015; 46 (4): $887-$ 93. doi: 10.1183/13993003.00432-2015.

25.- DIACON AH, PYM A, GROBUSCH M, PATIENTIA R, RUSTOMJEE R, PAGE-SHIPP L, et al. The diarylquinoline TMC207 for multidrug-resistant tuberculosis. N Engl J Med. 2009; 360: 2397-405.

26.- DIACON AH, PYM A, GROBUSCH MP, DE LOS RIOS JM, GOTUZZO E, VASILYEVA I, et al.Multidrug-resistant tuberculosis and culture conversion with bedaquiline. N Engl J Med. 2014; 371: 72332. 
27.- GLER MT, SKRIPCONOKA V, SÁNCHEZGARAVITO E, XIAO H, CABRERA-RIVERO JL, VARGAS-VÁSQUEZ D, et al. Delamanid for multidrug-resistant pulmonary tuberculosis. N Engl J Med. 2012; 366: 2151-60.

28.- HAFKIN J, HITTEL N, MARTIN A, GUPTA R. Early outcomes in MDR-TB and XDR-TB patients treated with delamanid under compassionate use. Eur Respir J. 2017; 50: 1700311.

29.- GUPTA R, GEITER LJ, WELLS CD, GAO M, CIRULE A, XIAO H. Delamanid for extensively drug resistant tuberculosis. N Engl J Med. 2015; 373: 291-2.

30.- MINISTERIO DE SALUD DE CHILE. Informes de situación epidemiológica y operacional del Programa Nacional de Tuberculosis de Chile, 2011-2019. PROCET. Material técnico histórico. www.minsal.cl

31.- ZÚÑIGA A, DAZA P. Ordinario B21 No 4276 del
7-10-2020. Subsecretaría de Salud Pública y Subsecretaría de Redes Asistenciales. Ministerio de Salud de Chile.

32.- XPERT MTB-XDR. www.cepheid.com. For information only: 301-1404-ES, Rev. F Agosto de 2019.

33.- WHO operational handbook on tuberculosis. Module 4: treatment - drug-resistant tuberculosis treatment. Geneva: World Health Organization; 2020. Licence: CC BY-NC-SA 3.0 IGO.

34.- TWEED CD, DAWSON R, BURGER DA, CONRADIE A, CROOK AM, MENDEL CM, et al. Bedaquiline, moxifloxacin, pretomanid, and pyrazinamide during the first 8 weeks of treatment of patients with drugsusceptible or drug-resistant pulmonary tuberculosis: a multicentre, open-label, partially randomised, phase $2 \mathrm{~b}$ trial. Lancet Respir Med. 2019; 7: 1048-58. Disponible en: https://doi.org/10.1016/S2213-2600(19)30366-2.

Correspondencia a:

Dr. Carlos Peña Mantinetti

Encargado del PROCET,

Servicio de Salud Metropolitano Central.

Hospital Clínico San Borja Arriarán. Santiago, Chile.

Email: carpemanti@hotmail.com 